\title{
Duodenal Gastrointestinal Stromal Tumor: A Rare Disease in a Young Adult Female Patient Presenting with Life-Threatening Hemorrhage
}

\author{
Manrica Fabbi Laura Castoldi Maurizio Sallusti Giorgio Rossi \\ Paolo Reggiani
}

Fondazione IRCCS Cà Granda, Maggiore Policlinico Hospital, Milan, Italy

\section{Keywords}

Bleeding · Duodenal gastrointestinal stromal tumors - Life-threatening acute hemorrhage ·

Young patient

\begin{abstract}
Duodenal gastrointestinal stromal tumors (dGISTs) may be a source of life-threatening hemorrhage that leads to emergency surgical care, precluding tumor staging and the planning of an elective treatment. In this study, we report a case of potentially lethal bleeding dGIST in a young woman successfully treated by an organ-preserving elective surgery after endoscopic and angiographic hemostasis. A 26-year-old female patient was admitted to the Emergency Unit of our hospital with the complaints of hematemesis and melena in the previous $12 \mathrm{~h}$. An upper endoscopy showed a 4-cm submucosal lesion, between the 2nd and 3rd part of the duodenum, in the lateral wall, with massive bleeding arising from central ulceration. Hemostasis was initially achieved endoscopically and then optimized by transarterial embolization. After a contrast-enhanced $\mathrm{CT}$, the patient underwent planning elective surgery. Intraoperatively, a 3-cm lesion was confirmed and resected by excision of the full-thickness duodenum with adequate free margins. Immunohistochemical analysis of the specimen revealed to be a dGIST, with a low mitotic count ( $<5$ mitosis/50 high power field), and tumor necrosis present in $<50 \%$ of the lesion. The patient had an uneventful course.
\end{abstract}


Fabbi et al.: dGIST in a Young Adult Female

\section{Introduction}

Gastrointestinal stromal tumors (GISTs) are the most common mesenchymal neoplasms of the gastrointestinal tract [1]. GISTs may occur anywhere in the digestive tract, but often located in the stomach $(60 \%)$ or small intestine (35\%), while the colon, rectum, appendix (5\%), and esophagus (2-3\%) are relatively rare sites. Duodenal GISTs constitute $<5 \%$ of all GISTs and represent up to $30 \%$ of all primary malignant duodenal tumors. They are generally manifested in adults over 50 years, mainly male, and exceptionally in children or young adults. The clinical presentations are highly variable and related to the origin, growth (intramural or extramural), and size [2]. The pathophysiology or surgical treatment of duodenal GISTs poses particular challenges for either diagnosis or management. GIST presentation with acute hemorrhage could lead to emergency surgical care, precluding the possibility of tumor staging and the planning of a more thoughtful elective treatment. In this study, a case of bleeding duodenal GIST with life-threatening hemorrhage was described in a 26-year-old woman, located in the 2nd-3rd duodenal tract with a 4-cm size, who was admitted in hemorrhagic shock due to gastrointestinal bleeding. The case was successfully treated by an organpreserving elective surgery after endoscopic and angiographic hemostasis. Presentation, diagnosis, and the type of performed treatment are reported and discussed in light of the available literature on duodenal GIST-related issues.

\section{Case Report}

A 26-year-old female patient was admitted to the Emergency Unit of the Maggiore Policlinico Hospital (Milan, Italy) with the complaints of hematemesis and melena in the previous $12 \mathrm{~h}$. She denied fever or weight loss, with no history of previous medical problem. On clinical examination, she was pale with a pulse rate of $125 / \mathrm{min}$, blood pressure $75 / 50 \mathrm{mmHg}$, and Glasgow Come Scale 13/15. A digital rectal examination confirmed melena. Her hemoglobin level was $7.1 \mathrm{~g} / \mathrm{dL}$. After fluid resuscitation and 2 units of red blood cell transfusions, an emergency superior digestive endoscopy was performed. It showed a $4-\mathrm{cm}$ submucosal lesion located between the 2nd and 3rd part of the duodenum, in the lateral wall. This mass had a central ulceration of $4 \mathrm{~mm}$, which was the origin of the massive bleeding (shown in Fig. 1a). In an attempt to perform a biopsy, the lesion started bleeding again. Primary hemostasis was performed endoscopically by the injection of a saline-diluted adrenalin and the application of 2 hemoclips (shown in Fig. 1b). After local hemostasis, tumor staging was initiated to plan further treatment strategies. Contrast-enhanced CT scan showed a well-defined hypervascular solid lesion of the duodenum, with dense peripheral vascularization in the arterial phase and heterogeneous contrast enhancement due to central necrosis (shown in Fig. 2a). Tortuous feeder vessels seemed to arise from the gastroduodenal artery. No distant metastases were highlighted. Based on lesion characteristics, a primary GIST of the duodenum was suspected, and the patient, with stable vital parameters, was transferred to the Emergency Surgery ward. During overnight observation, a new episode of melena caused a deterioration of patient status; hemodynamic parameters become unstable, and hemoglobin level dropped to $5.4 \mathrm{~g} / \mathrm{dL}$, requiring fluid resuscitation and blood transfusions. Emergency angiography showed an abnormal lesion vascularization arising from branches of the superior mesenteric artery. Transarterial embolization of these vessels was performed, using absorbable hemostatic gelatin sponge and metallic coils (shown in Fig. 2b, c). No further bleeding was detected over the course of the next $48 \mathrm{~h}$. Hyperenhancement of the lesion and chromogranin A level $(126 \mu \mathrm{g} / \mathrm{L}$, slightly higher compared to the normal level of $100 \mu \mathrm{g} / \mathrm{L})$ suggested the possibility of a neuroendocrine tumor. After planning an elective surgical resection, the patient was

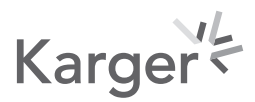



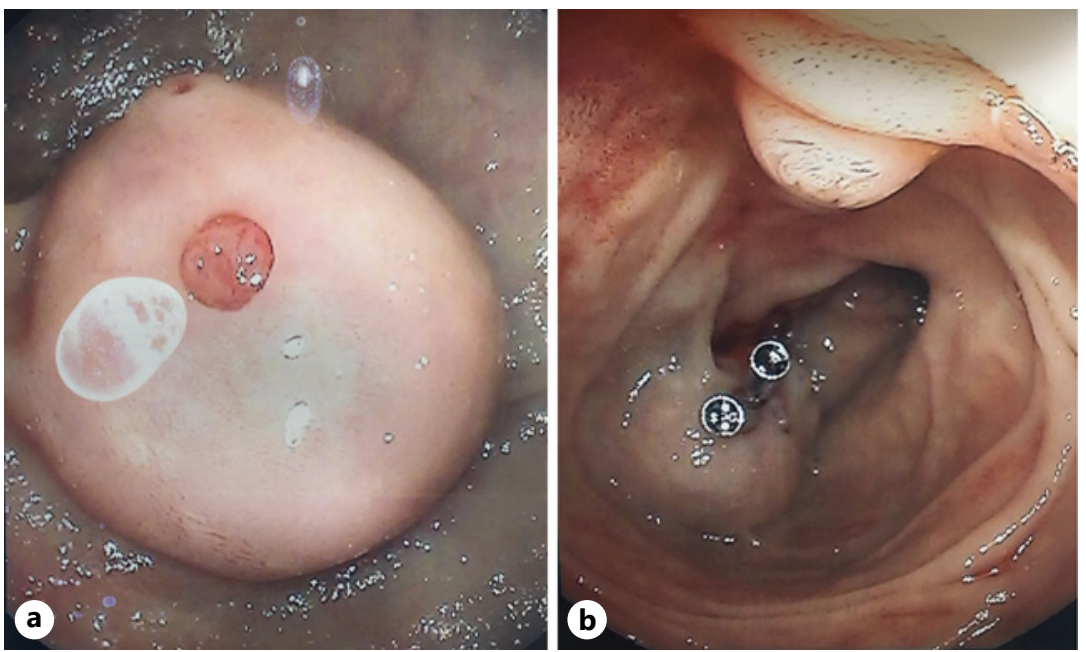

Fig. 1. Submucosal lesion protruding into the duodenal lumen, with central mucosa ulceration (a); application of 2 hemoclips on the ulcerated lesion (b).
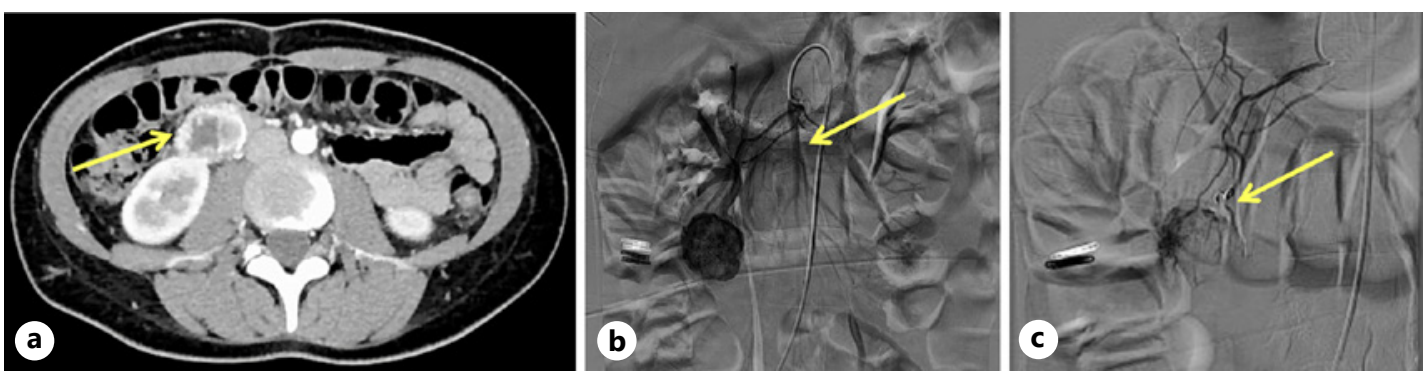

Fig. 2. CT scan showing a lesion (arrow) arising from the 2nd-3rd part of the duodenum, with peripheral vascularity and heterogeneous central enhancement due to the colliquative necrotic components (a); lesion vascularization arising from branches of the SMA (b); angiogram showing successful coil embolization of distal selected branches of the SMA (c). SMA, superior mesenteric artery.

operated. After laparotomy and exploration of the peritoneal cavity, a 3-cm tumor was found surrounded by multiple vessels (shown in Fig. 3). Cut section of the duodenum revealed an encapsulated mass in the lateral wall of the duodenum with a predominant exophytic component and ulcer in the medial wall of the lesion. The histopathological exam of the frozen section revealed features of submucosal GIST. Therefore, GIST was excised entirely by a limited resection of the duodenum wall and duodenotomy closed by the hand-sewn doublelayer sutures technique (shown in Fig. 3). The postoperative course was uneventful, and the gastrointestinal oral contrast study confirms anastomosis integrity. The patient was discharged from the hospital on the 9th postoperative day. The final histological examination of the specimen revealed mesenchymal tumor tissue within the submucosa, with a clear resected margin. The tumor was positive for CD117 (C-KIT) and DOG1 and negative for CD34 and desmin. The lesion displayed a low mitotic count $(<5$ mitosis $/ 50$ high power field) and tumor necrosis present in $<50 \%$ of the lesion (shown in Fig. 4). The tumor was diagnosed as a GIST: TNM 8th pt2. These findings correspond to a prognostic group 2 (WHO classification, 2010), low risk according to Miettinen et al. [3]. 


\section{Case Reports in Gastroenterology}

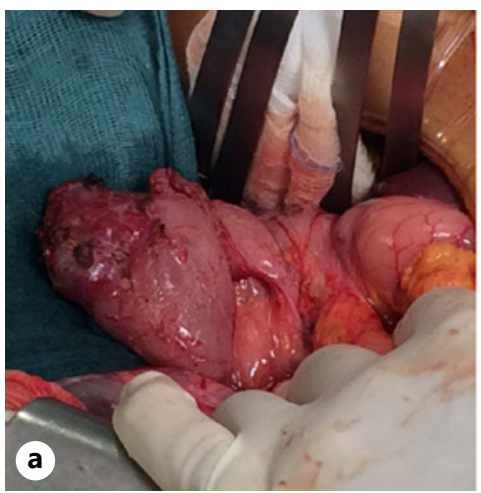

\begin{tabular}{l|l}
\hline Case Reports in Gastroenterology \\
\hline DOI: 10.1159/000515370 & $\begin{array}{l}\text { @ 2021 The Author(s). Published by S. Karger AG, Basel } \\
\text { www.karger.com/crg }\end{array}$ \\
\hline
\end{tabular}

Fabbi et al.: dGIST in a Young Adult Female

Fig. 3. GIST of the 2nd-3rd duodenal portion (a); duodenal wall after GIST removal (b); duodenotomy by double-layer hand-sewn sutures (c). GIST, gastrointestinal stromal tumor.
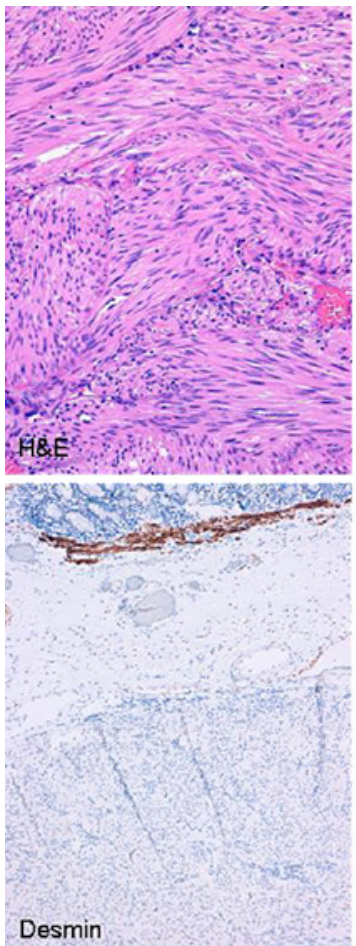
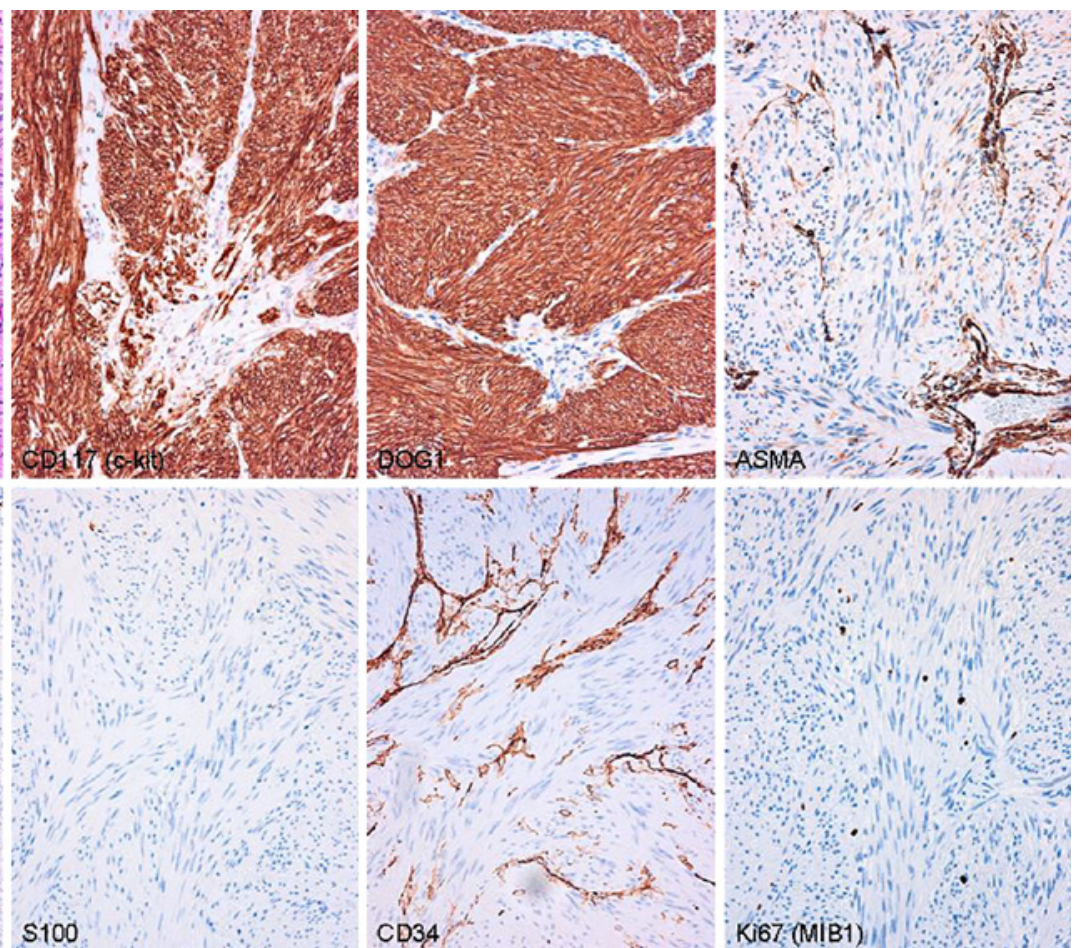

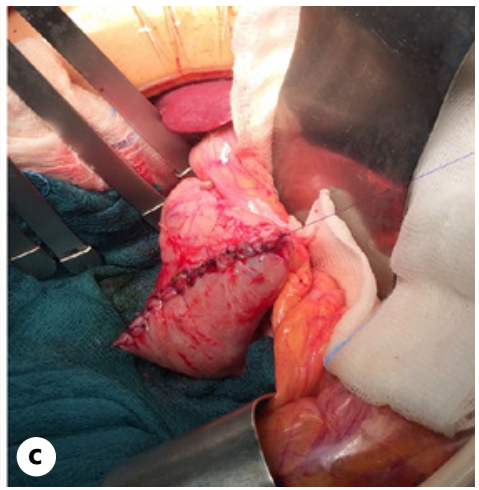

Fig. 4. Tumor was constituted by spindle cells with low nuclear pleomorphism, organized in storiform fascicles $(H \& E, \times 200)$; tumor cells were strongly immunoreactive for c-kit $(\times 200)$ and DOG1 $(\times 200)$ and focally weakly positive for smooth muscle actin $(\times 200)$; negative staining was observed for desmin $(\times 100)$, S110 $(\times 200)$, and CD34 $(\times 200)$. A low proliferative index was demonstrated by Ki67 staining $(\times 200)$.

\section{Discussion and Conclusion}

The duodenum represents a very rare site of primary GIST, with an incidence $<5 \%$ of all cases $[1,3]$. Duodenal GIST is typically observed in adults (50-70 years) with a slight preponderance of males. Children or young adults are sporadically present in multipatient GIST studies. Most relevant case studies about GISTs with severe bleeding under 30-yearsold reported male patients with age between 7 and 29, except for a female of 19 [4]. In this report, a rare case of a duodenal GIST was described in a 26-year-old female patient manifesting 
with acute life-threatening hemorrhage with consecutive hemorrhagic shock. Although duodenal GISTs are often asymptomatic, particularly when of small size, in symptomatic patients, gastrointestinal bleeding is a frequent presentation of these tumors [5]. The cause of hemorrhage can be mucosal ulceration at peritumoral submucosal large vessels or, as in our patient, at the tumor dome. This type of manifestation in young patients has been mainly treated by urgent surgery in the reported cases. In early reports [6, 7], this procedure was justified by the unsuccessful attempts of identifying the active source of bleeding through upper-GI endoscopy and arteriography, while in the study by Mokhtare et al. [8] and Elston et al. [9], endoscopy has been only adopted for diagnostic purpose. The presence of massive bleeding tends to prioritize the life-saving procedure to block hemorrhage over other diagnostic or therapeutic modalities. However, this may incur in a second intervention, as by Mennigen et al. [10], in which the failure of preoperative hemostasis has required a first urgent surgery to control bleeding prior to the tumor removal. As previously reported by Valli et al. [11] and in our case, repeated angiographies had allowed the halting of hemorrhage by transarterial embolization and the conversion of a surgical emergency into an elective procedure. Apart from reducing urgent surgical related-complication [12], patient stabilization allows the eventual application for a neoadjuvant treatment, particularly relevant in case of large tumor size and/or pancreas involvement. The improvement of devices (i.e., hemostatic powders and forceps, OTSCs, and other emerging technologies) along with operator's skills has increased the success rate of preoperative hemostasis [13], which, in our case, was decisive for patient stabilization. Optimal surgical strategies for duodenal GISTs remain to be established. The lack of a consensus depends on the relatively low incidence and limited literature evidence. Also, the anatomy of the duodenum, especially the 2 nd part, and its proximity to important anatomical structures, such as the duodenal papilla, pancreas, and biliary and pancreatic ducts, further complicate this task $[2,10]$. Treatment options range from pancreatoduodenectomy to local resection, mostly depending to lesion size and location $[14,15]$. For a small tumor size and localized in the lateral wall of the 2 nd-3rd duodenal portion, local resection is generally recommended, without clear guidelines between wedge or segmental resection [14]. In our case, given a tumor size of about $3 \mathrm{~cm}$, a wedge resection was opted, performing a primary closure instead of a Roux-en-Y duodenojejunostomy. While the 2 types of surgical reconstruction are often considered interchangeable and chosen based on surgeon preference, complications has been sometimes reported for the Roux-en-Y duodenojejunostomy. However, evidence from an in-depth comparison is still lacking in the literature. In conclusion, we reported a rare case of duodenal GIST in a young patient successfully managed through preoperative stabilization and elective surgery. In our view, endoscopy or arteriography embolization should be primarily pursued, along with a surgical treatment with lower morbidity but comparable oncological outcomes.

\section{Acknowledgment}

We thank Dr. Maggioni Marco (Fondazione IRCCS Cà Granda, Maggiore Policlinico Hospital, Milan, Italy) for providing the histological images of the specimen.

\section{Statement of Ethics}

Written informed consent was obtained from the patient to publish the case (including images).

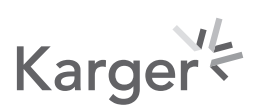




\section{Conflict of Interest Statement}

The authors have no conflicts of interest to declare.

\section{Funding Sources}

This work has been partially funded by Associazione Italiana Copev.

\section{Author Contributions}

Fabbi M. participated in data collection, writing the manuscript, and literature review and finalized the final draft of the manuscript; Castoldi L. participated in collecting data and coordination and reviewed the final draft of the manuscript. Sallusti M., Reggiani P., and Rossi G. reviewed the final draft of the manuscript. All authors read and approved the final manuscript.

\section{References}

1 Rubin BP, Heinrich MC, Corless CL. Gastrointestinal stromal tumour. Lancet. 2007;369(9574):1731-41.

2 Chung JC, Chu CW, Cho GS, Shin EJ, Lim CW, Kim HC, et al. Management and outcome of gastrointestinal stromal tumors of the duodenum. J Gastrointest Surg. 2010;14(5):880-3.

3 Miettinen M, Lasota J. Gastrointestinal stromal tumors: pathology and prognosis at different sites. Semin Diagn Pathol. 2006;23(2):70-83.

4 Popivanov G, Tabakov M, Mantese G, Cirocchi R, Piccinini I, D'Andrea V, et al. Surgical treatment of gastrointestinal stromal tumors of the duodenum: a literature review. Transl Gastroenterol Hepatol. 2018;3:71.

5 Sorour MA, Kassem MI, Ghazal Ael-H, El-Riwini MT, Abu Nasr A. Gastrointestinal stromal tumors (GIST) related emergencies. Int J Surg. 2014;12(4):269-80.

6 Towu E, Stanton M. Gastrointestinal stromal tumour presenting with severe bleeding: a review of the molecular biology. Pediatr Surg Int. 2006;22(5):462.

7 Chiarugi M, Galatioto C, Lippolis P, Zocco G, Seccia M. Gastrointestinal stromal tumour of the duodenum in childhood: a rare case report. BMC Cancer. 2007;7:79.

8 Mokhtare M, Taghvaei T, Tirgar Fakheri H. Acute bleeding in duodenal gastrointestinal stromal tumor. Middle East J Dig Dis. 2013 Jan;5(1):47-51.

9 Elston MS, Sehgal S, Dray M, Phillips E, Conaglen JV, Clifton-Bligh RJ, et al. A duodenal SDH-deficient gastrointestinal stromal tumor in a patient with a germline sdhb mutation. J Clin Endocrinol Metab. 2017;102(5):1447.

10 Mennigen R, Wolters HH, Schulte B, Pelster FW. Segmental resection of the duodenum for gastrointestinal stromal tumor (GIST). World J Surg Oncol. 2008;6:105.

11 Valli P, Valli C, Pfammatter T, Bauerfeind P. Life-threatening bleeding of a duodenal gastrointestinal stromal tumor in a teenager: a rare case report. Endosc Int Open. 2016;4(12):E1244-46.

12 Mullen MG, Michaels AD, Mehaffey HJ, Guidry CA, Turrentine LE, Hedrick TL, et al. Risk associated with complications and mortality after urgent surgery vs elective and emergency surgery : implications for defining "quality" and reporting outcomes for urgent surgery. JAMA Surg. 2017;152:768-74.

13 Tarasconi A, Baiocchi GL, Pattonieri V, Perrone G, Abongwa HK, Molfino S, et al. Transcatheter arterial embolization versus surgery for refractory non-variceal upper gastrointestinal bleeding: a meta-analysis. World J Emerg Surg. 2019;14:3.

14 Cavallaro G, Polistena A, D’Ermo G, Pedullà G, De Toma G. Duodenal gastrointestinal stromal tumors: Review on clinical and surgical aspects. Int J Surg. 2012;10(9):463.

15 Chok AY, Koh YX, Ow MYL, Allen JC, Goh BKP. A systematic review and meta-analysis comparing pancreaticoduodenectomy versus limited resection for duodenal gastrointestinal stromal tumors. Ann Surg Oncol. 2014; 21(11):3429-38.

\section{Karger'}

